MATEC Web of Conferences 52, 02005 (2016)

DOI: $10.1051 /$ matecconf/20165202005

C Owned by the authors, published by EDP Sciences, 2016

\title{
Effect of Local Constraint on Driving Torque of Driving Mechanism for Half-rotating Wing
}

\author{
Huixing Wang, Xiaoyi Wang ${ }^{\mathrm{a}}$, Han Qiu, Fuqiang Chen, Zhizhen Qiu and Jianhua Shan \\ School of Mechanical Engineering, Anhui University of Technology, Ma'anshan, 243002, P.R.China
}

\begin{abstract}
Half-rotating wing (HRW) is a new kind of flapping wing system with rotating-type flapping instead of oscillating-type flapping. For the complex mechanical transmission and high weight which is not suitable for flight of traditional half-rotating mechanism (HRM).The simplified HRM with local constraint was proposed as the driving mechanism of HRW in this paper. The performing process and characteristics of local constraint was further given. According to contact state between moving components, the performing process of local constraint was divided into two stages. To analyze the influence of local constraint on the driving torque exerted in the crank of driving mechanism, the model of driving torque under different stages were respectively established. Based on the model, the change curve of driving torque was obtained through simulation by MATLAB, which showed that local constraint had little influence on driving torque. The results could provide guidance for the choice of motor of HRW and optimization of dynamics.
\end{abstract}

\section{Introduction}

Flapping-wing air vehicle had become research hotspot due to its excellent maneuverability and hovering ability beyond traditional fixed wing and micro rotary wing[1].Researchers superimposed two same sets of crank-rocker mechanisms to create the Micro Bat[2].Madangopal et al designed the Sparrow by combining slider-crank mechanism with double-slider mechanism[3].Wei Zhen et al invented the parallel crank-rocker flapping mechanism[4].However, these mechanisms would produce huge inertia force due to high frequency oscillation, which limited its operating frequency and the scope of work.

During the study of bionic machinery, it could be found that animals' movement was essentially asymmetric. A kind of single degree mechanism named HRM was proposed as the driving mechanism for HRW, which could achieve flapping just by asymmetric rotating. However, HRM driven by gear or chain[5-7] was not suitable for flight due to the complex mechanical transmission and high weight for which the simplified design was needed. To ensure that the movement of HRM is accurate in a given time, a temporary constraint, called local constraint, was added artificially in the process of simplification. On the other hand, torque on the driving mechanism would be changed when local constraint worked with and without. Therefore, mechanical model was established and change of driving torque was determined. Both of these were guidance for the choice of motor of HRW and optimization of dynamics.

\footnotetext{
${ }^{\mathrm{a}}$ Corresponding author : wangxy@ahut.edu.cn
} 


\section{Driving mechanism of HRW and local constraint}

\subsection{Driving mechanism of HRW}

The sketch of HRW was shown in Fig.1(a), whose driving mechanism was designed based on the principle of HRM. HRM was consist of crank and half-rotating rod, as shown in Fig.1(b).Half-rotating rod would turn half round anticlockwise when crank turned one round anticlockwise. However, it was hard to realize the moving rule of HRM according to this concept for the mechanism would interfere when rotation center $C$ moved to stagnation point $H$. Therefore, one edge of the crank was designed as shaft with groove. Furthermore, positioning slider was set at the location of point $H$ to make sure HRM had accurate moving rule. The combination of positioning slider and shaft with groove could realize simplified design of HRM in Fig.1(c). Herein, point $C$ was the center of shaft, point $H$ denoted the rotation center of positioning slider.

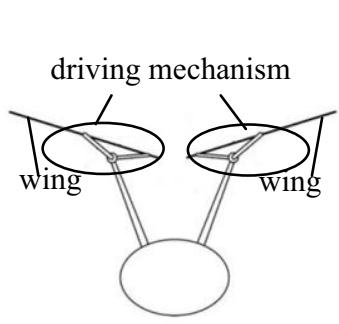

(a) Aircraft using HRM

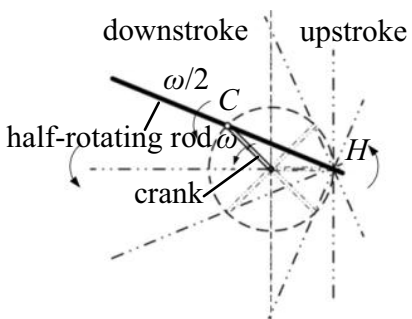

(b) Principle of HRM

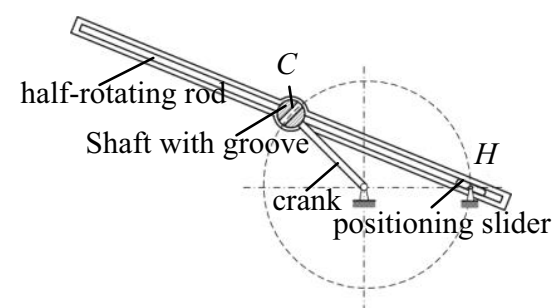

(c) The simplified HRM

Figure 1. HRW aircraft and its driving mechanism

\subsection{Working principle of local constraint}

In the movement of driving mechanism of HRW, when the center of positioning slider was not in shaft with groove, the half-rotating rod would rotate according to half-rotating law with constraint from the center of positioning slider. After center of positioning slider entered the groove of shaft, as shown in Fig.2(a), positioning slider could not rotate freely due to the constraint from arc-shaped groove at the interior of groove. Meanwhile, half-rotating rod would rotate with positioning slider due to the constraint at the exterior of groove. This constraint made up of positioning slider, shaft with groove and half-rotating rod, functioned transiently and locally, which was called local constraint. Fig.2 showed the relative position of positioning slider, shaft with groove and half-rotating rod with local constraint at different time.

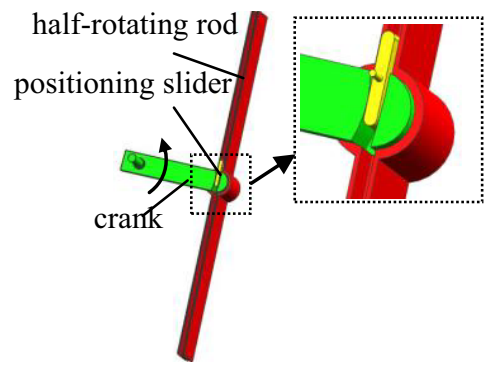

(a) The beginning of local constraint

Figure 2. Local constraint at different time

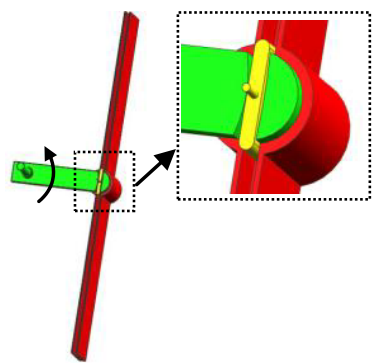

(b) In the middle of local constraint

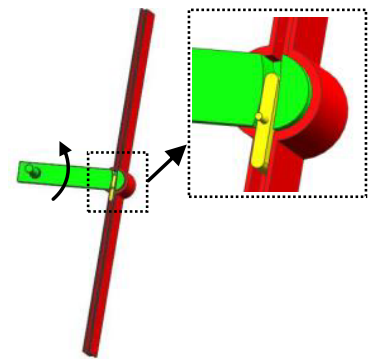

(c) At the end of local constraint 
Positioning slider would not affect the movement of half-rotating rod without local constraint. Fig. 3 showed the simplified model of driving mechanism for HRW without local constraint. Herein, $O C$ denoted crank, $A B$ represented half-rotating rod. Blade of HRW and half rotation rod were fixed. The force from air on the blade was a distributed force system, which could be simplified as principal vector $F_{\mathrm{R}}$ and principal moment $M_{\mathrm{C}}$ at any instantaneous. The force on crank could be decomposed into $X_{\mathrm{O}}$ horizontal and $Y_{\mathrm{O}}$ vertical directions.

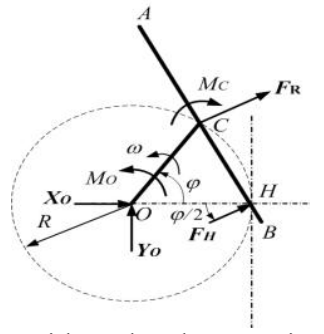

Figure 3. Force analysis of driving mechanism without local constraint

Model of driving torque $M_{\mathrm{o}}$ could be calculated by combining $\sum M_{O}=0$ with $\sum M_{C}=0$.

$$
M_{O}=\frac{1}{32} C_{D} \rho h \omega^{2}\left(16 R^{4} \sin ^{4} \frac{\varphi}{2}+24 R^{2} a^{2} \sin ^{2} \frac{\varphi}{2}+a^{4}\right)
$$

Where $C_{\mathrm{D}}$ was drag coefficient of blade which could be obtained by experiment. $\rho$ was air density. $h$ was width of blade. $a$ was length of blade. $R$ was length of crank. $\omega$ was angular velocity of crank.

\section{Model of driving torque on driving mechanism for HRW with local constraint}

\subsection{Different stages under local constraint}

Inside contact stage was the first stage, which was started from the beginning of local constraint to the coincidence of point $C$ and point $H$. The relevant components of driving mechanism under this stage was shown in Fig. 4(a). Herein, $F_{1}$ was the force on positioning slider excited by half-rotating rod, $F_{2}$ was the force on positioning slider excited by the inside arc of shaft with groove, $F_{\mathrm{H}}$ denoted the constraining force on positioning slider shaft excited by bracket. It could be seen clearly that, the inside surface of positioning slider and half-rotating rod were contacted with each other, and the inside surface of positioning slider contacted with the inside arc of shaft with groove.

Outside contact stage was the second stage, which started from the separation of point $C$ and point $H$ to the end of local constraint. The relevant components of driving mechanism under this stage was shown in Fig. 4(b). Herein, $F_{1}$ was the force on positioning slider excited by half rotating rod, $F_{2}$ was the force on positioning slider excited by the outside arc of shaft with groove, $F_{\mathrm{H}}$ was the constraining force on positioning slider shaft excited by bracket. it could be found from Fig. 4(b) that the outside surface of positioning slider and half-rotation rod were contacted with each other, and the outside surface of positioning slider contacted with the outside arc of shaft with groove.

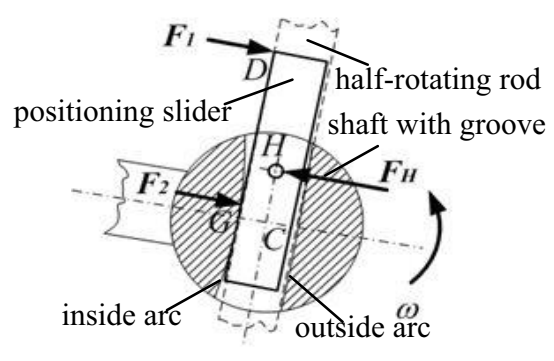

(a) Inside contact stage

Figure 4. Different stages of local constraint

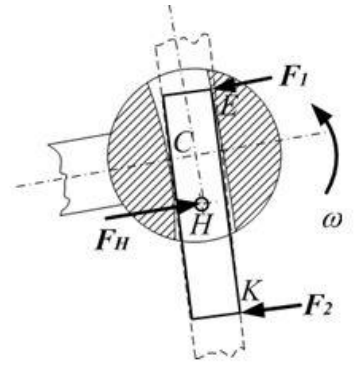

(b) Outside contact stage 


\subsection{Driving torque model under different stages}

This article was focused on the calculation of the driving torque under inside contact stage. As space is limited, other similar derivation was omitted, and the results were given directly.

\subsubsection{Driving torque model under inside contact stage}

The contact state of driving mechanism was shown in Fig.4(a). The force $F_{2}$, which passed through the center of inner arc, was closely perpendicular to the half-rotating rod. So the force system acting on the positioning slider could be regarded as a plane parallel force system.

(1) Firstly, half-rotating rod was regarded as research object and force analysis was shown in Fig.5

The groove of half-rotating rod and positioning slider were represented by solid line and dotted line respectively. $F_{1}^{\prime}$ was the force on half-rotating rod excited by the positioning slider and it could be calculated as follow.

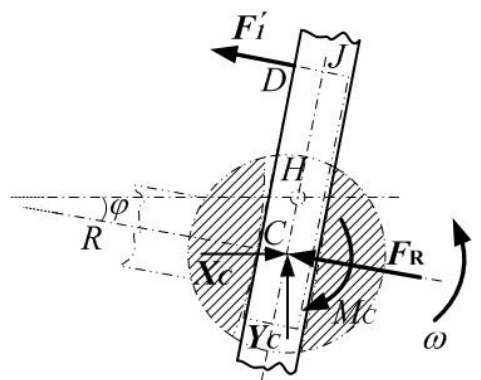

Figure 5. Force analysis of half-rotating rod under inside contact stage

$$
F_{1}^{\prime}=\frac{M_{C}}{2 R \sin \frac{\varphi}{2}+b}
$$

Where $b$ was half the length of the positioning slider and $M_{\mathrm{C}}$ could be obtained from literature [5].

(2) secondly, positioning slider was regarded as research object and force analysis was shown in Fig.6

Herein, line $H E$, which was tangent with circle $O_{2}$ at point $E$, was parallel to line $D G$. On the other hand, $F_{2}$ was perpendicular to line $D G$. So $H E$ was the arm of $F_{2}$.

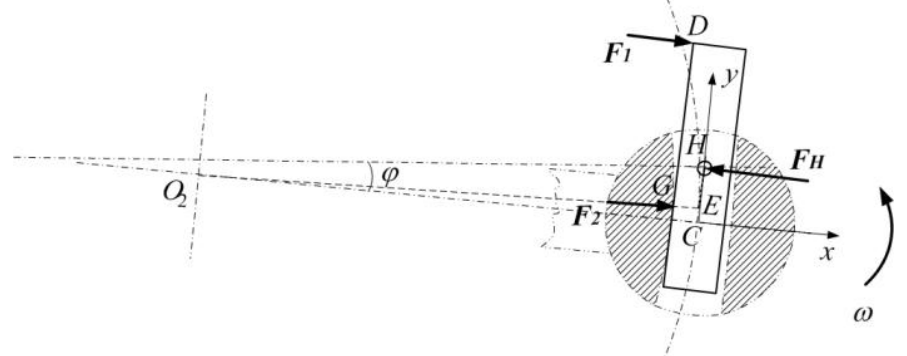

Figure 6. Force analysis of positioning slider under inside contact stage

$F_{\mathrm{H}}$ could be calculated by equations (2), $\sum M_{H}=0$ and $\sum F_{x}=0$.

$$
F_{H}=\frac{M_{C}\left(2 R \sin \frac{\varphi}{2}+\sqrt{b^{2}+r}\right)}{4 R^{2} \sin ^{2} \frac{\varphi}{2}+2 R b \sin \frac{\varphi}{2}}
$$

(3) Finally taking the driving mechanism as the research object.

Driving torque $M_{\mathrm{O}}$ could be obtained by combining Eq.(3) and equation $\sum M_{O}=0$. 


$$
M_{O}=\frac{1}{48} C_{D} \rho h \omega^{2}\left((32-16 A) R^{4} \sin ^{4} \frac{\varphi}{2}+24(\mathrm{~A}+1) a^{2} R^{2} \sin ^{2} \frac{\varphi}{2}+3 A a^{4}\right)
$$

where $A=\frac{2 R \sin \frac{\varphi}{2}+2 b-\sqrt{b^{2}+r}}{4 R \sin \frac{\varphi}{2}+2 b}$ and $r$ was radius of shaft with groove.

\subsubsection{The driving torque model under outside contact stage}

The driving torque model under outside contact stage could be expressed as:

$$
M_{O}=\frac{1}{48} C_{D} \rho h \omega^{2}\left((32-16 B) R^{4} \sin ^{4} \frac{\varphi}{2}+24(\mathrm{~B}+1) \mathrm{a}^{2} R^{2} \sin ^{2} \frac{\varphi}{2}+3 B \mathrm{a}^{4}\right)
$$

where $B=\frac{b}{2 R \sin \frac{\varphi}{2}+b}$.

\section{Simulation analysis of driving torque on driving mechanism}

Table 1. Calculation parameters of driving torque on driving mechanism of HRW

\begin{tabular}{cc}
\hline Calculation parameters & Size \\
Lift coefficient $C D$ & 4.6 \\
Air density $\rho\left(\mathrm{kg} \cdot \mathrm{m}^{-3}\right)$ & 1.225 \\
Width of blade $h(\mathrm{~m})$ & 0.2 \\
Length of blade $a(\mathrm{~m})$ & 0.141 \\
Length of crank $R(\mathrm{~m})$ & 0.06 \\
Length of slider $2 b(\mathrm{~m})$ & 0.02 \\
Radius of shaft $r(\mathrm{~m})$ & 0.008 \\
\hline
\end{tabular}

For the convenience of analysis, the initial position of the crank was assumed to be left. Since the angular velocity of crank was $360 \%$, drive mechanism could run a circle with $1 \mathrm{~s}$. Among them, the stage without local constraint, inside contact stage and outside contact stage were $0.96 \mathrm{~s}, 0.02 \mathrm{~s}$ and $0.02 \mathrm{~s}$. Then Matlab was used to calculated the driving torque's equations (1), (4), (5) with HRW prototype size and related experimental data, as shown in table 1. Finally, we could use Matlab simulated the running process of driving mechanism and the curve of driving torque was obtained, as shown in Fig.7.

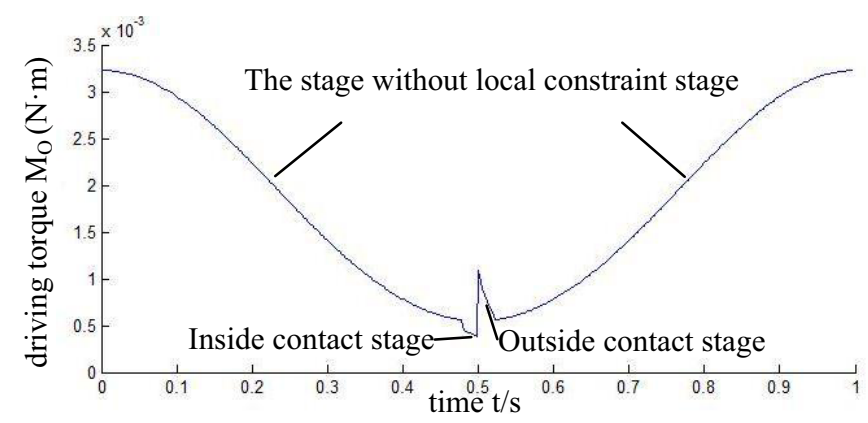

Figure 7 .Driving torque curve on driving mechanism for HRW 
Fig.7 showed the driving torque curve of driving mechanism for HRW in one cycle. It could be found that the driving torque uniformly changed over time under the stage without local constraint, but when from the stage without local constraint stage to inside contact stage and the stage from inside contact to outside contact, the driving torque mutation occurred, and the latter was more obvious. The curve could be proved that the local constraint applied on the HRW had influence on driving torque, but not very seriously.

\section{Conclusion}

(1)Local constraint and its application in the simplified HRM made driving mechanism of HRW more simple in structure and lighter in weight, and the improving of lift-to-weight ratio was more conducive to flying as well.

(2)For the different contact stages of driving mechanism under local constraint, the driving torque model was established. On the basis of model, the curve of the driving torque during operating period were obtained through Matlab, which showed that local constraint had little influence on driving torque. All the work done in this stage lay down the solid foundations for the choosing of Motor and optimization of dynamics.

\section{Acknowledgments}

This work is supported by the National Natural Science Foundation of China (Grant No. 51375014 and No. 51405001)

\section{References}

1. R.J. Wood, IEEE.Trans.Rob. Autom, 24,341 (2004)

2. T.N.Pornsin-Sirirak,S.W.Lee.AICMEMS,799 (2000)

3. R. Madangopal, Z.A. Khan, S.K. Agrawal, J. Mech. Design,127,809,(2005)

4. Z. Wei, L.C.Jia, J.M. Yang. Mech. Eng ,33,63,(2011)

5. Z,Z, Qiu, Press.USTC,27(2011)

6. X.X Wang, L.L. Mei, F.Q.Chen,Mech.Sci.Tech.Aerosp.Eng, 30,1020(2011)

7. X.X. Wang, J.H. Qin, H. Qiu, Mech.Sci.Tech.Aerosp.Eng ,33,802 (2014) 